ARTICLE

DOI: $10.1038 / \mathrm{s} 41467-018-07502-5$

\title{
Two-dimensional transition metal carbides as supports for tuning the chemistry of catalytic nanoparticles
}

Zhe Li (1) ${ }^{1}$, Liang $\mathrm{Yu}^{2}$, Cory Milligan ${ }^{3}$, Tao Ma ${ }^{4}{ }^{4}$, Lin Zhou ${ }^{4}$, Yanran Cui ${ }^{3}$, Zhiyuan $\mathrm{Qi}^{5}$, Nicole Libretto ${ }^{3}$, Biao $\mathrm{Xu}^{1}$, Junwei Luo ${ }^{2}$, Enzheng Shi ${ }^{1}$, Zhenwei $\mathrm{Wu}^{3,6}$, Hongliang $\mathrm{Xin}^{2}$, W. Nicholas Delgass ${ }^{3}$, Jeffrey T. Miller ${ }^{3} \&$ Yue $\mathrm{Wu}^{1}$

Supported nanoparticles are broadly employed in industrial catalytic processes, where the active sites can be tuned by metal-support interactions (MSIs). Although it is well accepted that supports can modify the chemistry of metal nanoparticles, systematic utilization of MSIs for achieving desired catalytic performance is still challenging. The developments of supports with appropriate chemical properties and identification of the resulting active sites are the main barriers. Here, we develop two-dimensional transition metal carbides (MXenes) supported platinum as efficient catalysts for light alkane dehydrogenations. Ordered $\mathrm{Pt}_{3} \mathrm{Ti}$ and surface $\mathrm{Pt}_{3} \mathrm{Nb}$ intermetallic compound nanoparticles are formed via reactive metal-support interactions on $\mathrm{Pt} / \mathrm{Ti}_{3} \mathrm{C}_{2} \mathrm{~T}_{x}$ and $\mathrm{Pt} / \mathrm{Nb}_{2} \mathrm{CT}_{x}$ catalysts, respectively. MXene supports modulate the nature of the active sites, making them highly selective toward $\mathrm{C}-\mathrm{H}$ activation. Such exploitation of the MSIs makes MXenes promising platforms with versatile chemical reactivity and tunability for facile design of supported intermetallic nanoparticles over a wide range of compositions and structures.

\footnotetext{
${ }^{1}$ Department of Chemical and Biological Engineering, lowa State University, Ames, IA 50011, USA. ${ }^{2}$ Department of Chemical Engineering, Virginia Polytechnic Institute and State University, Blacksburg, VA 24061, USA. ${ }^{3}$ Davison School of Chemical Engineering, Purdue University, West Lafayette, IN 47907, USA. ${ }^{4}$ Department of Energy, Ames Laboratory, Ames, IA 50011, USA. ${ }^{5}$ Department of Chemistry, lowa State University, Ames, IA 50011, USA. ${ }^{6}$ Present address: Department of Chemical and Engineering, Stanford University, Stanford, California 94305, USA. Correspondence and requests for materials should be addressed to Z.W. (email: zwwu@stanford.edu) or to H.X. (email: hxin@vt.edu) or to T.M. (email: mill1194@purdue.edu) or to Y.W. (email: yuewu@iastate.edu)
} 
$\mathrm{M}$ etal nanoparticles (NPs) are widely used as heterogeneous catalysts and in electrochemical applications ${ }^{1-}$ 3 . The performance of the supported metal NPs, including the rate, selectivity and stability, can be tailored by controlling their interactions with the supports ${ }^{4-7}$. These metalsupport interactions (MSIs) have been found to modify the geometric and electronic structures of active sites ${ }^{8,9}$, and, not surprisingly, the chemical properties of the supports are crucial to the modifications ${ }^{10}$. Nonetheless, rational design of supported catalysts through MSIs is often arduous, especially when the supports undergo structural changes under reaction conditions ${ }^{11}$. A classic example is the encapsulation of metal active sites by reducible oxide-support overlayers, which was designated by Tauster et al. ${ }^{12}$ as the strong-metal support interaction (SMSI) in 1978. In the SMSI state the active metal sites on the NPs are covered due to the migration of suboxide species, which renders the loss of adsorption capability of the NPs ${ }^{12,13}$. Supports with ideal chemical tunability and reactivity are clearly a key to harnessing the potential promotional effects of MSIs on supported metal NP catalysts, but their development remains a grand challenge.

Two-dimensional (2D) early transition metal carbides, i.e., MXenes, is a burgeoning class of materials with well-defined structures and widely tunable compositions ${ }^{14}$. They have a formula of $\mathrm{M}_{n+1} \mathrm{X}_{n} \mathrm{~T}_{x}$, where $\mathrm{M}$ is an early transition metal, $\mathrm{X}$ refers to carbon and/or nitrogen, and $\mathrm{T}$ stands for surface functional groups ${ }^{15}$. We have recently shown that MXenes are promising supports for nanoparticle (NPs) catalysts and that the presence of noble metal NPs promotes both the removal of surface functional groups and reduction of the M component of the MXene ${ }^{16}$. Reduction of these catalyst supports can lead to targeted delivery of the metal components in the supports to the NPs that contact the support surface. As a result, formation of ordered intermetallic compounds (IMCs) through reactive metal-support interactions (RMSIs) is possible ${ }^{17}$. RMSI refers to a chemical reaction between a metal and the support that induces the formation of bimetallic structures, which is differentiated from the more general SMSI because it is driven by the high thermodynamic stability of the resulting IMCs. MXenes can facilitate this process by having $2 \mathrm{D}$ structures with metal carbon bonds $(\mathrm{M}-\mathrm{C})$ that are weaker compared to the metal oxygen bonds $(\mathrm{M}-\mathrm{O})$ in typical oxide supports. This enhanced chemical reactivity can allow RMSIs to occur at lower temperature and, thus, favor the control of particle size, in contrast to the high temperature reduction required for early transition metal oxides or bulk carbides $^{18-20}$. On MXenes IMCs may be formed that are not possible on traditional oxide and carbide supports and their properties controlled through in situ reduction at moderate temperature.

Here, we report two examples of thermally stable intermetallic NP catalysts prepared via RMSI between platinum and MXenes. A complete, full $\mathrm{Pt}_{3} \mathrm{Ti}$ IMC with $\mathrm{Cu}_{3} \mathrm{Au}$ type structure is formed in $\mathrm{Pt} / \mathrm{Ti}_{3} \mathrm{C}_{2} \mathrm{~T}_{x}$ catalysts. For $\mathrm{Pt} / \mathrm{Nb}_{2} \mathrm{CT}_{x}$ catalysts, NPs with a surface $\mathrm{Pt}_{3} \mathrm{Nb}$ IMC in the same structure are formed, presumably via a process kinetically controlled by the diffusion of $\mathrm{Nb}$ species. These intermetallic structures have not been previously reported for Pt NPs catalysts supported by oxides and bulk carbides of Ti and $\mathrm{Nb}$. The strong intermetallic bonds in these structures offer compositional and electronic modification of the actives sites. The result, in this case is that the catalysts become highly selective for light alkane dehydrogenation (LADH), a reaction in renewed interest due to shale gas boom ${ }^{21}$. Such reactive interaction is generally applicable between platinum NPs and MXene families. The Pt-M (M refers to early transition metals) IMCs are famous for their thermal stability with high enthalpy of formation, but their preparation through co-reduction is challenging as early transition metals are oxyphilic ${ }^{22,23}$. Thus, MXenes pave an avenue for facile design of Pt-M NPs with a broad range of compositions and structures that are intractable to synthesize by traditional methods.

\section{Results}

Two-dimensionality of the MXene supports. The two MXene supports, $\mathrm{Ti}_{3} \mathrm{C}_{2} \mathrm{~T}_{x}$ and $\mathrm{Nb}_{2} \mathrm{CT}_{x}$, were prepared by a chemical exfoliation process reported by the literature ${ }^{14,24}$. Briefly, $\mathrm{Ti}_{3} \mathrm{AlC}_{2}$ and $\mathrm{Nb}_{2} \mathrm{AlC}$ compounds (MAX) were treated with HF to extract the aluminum layers and exfoliate the $2 \mathrm{D}$ early transition metal carbides (Fig. 1a). In the X-ray diffraction (XRD) patterns (Supplementary Figs. 1a, b), the shift of (002) peaks and the disappearance of the most intense nonbasal plane diffraction peaks of the MAX phases at $2 \theta \approx 39^{\circ}$ indicate that the MAX phases are converted to MXenes with increased c lattice parameters after the HF exfoliation. The scanning electron microscopy (SEM) images (Fig. 1b, Supplementary Fig. 1c) display the typical accordion-like morphology of MXenes that suggests the exfoliation of individual grains along the basal planes. Dimethyl sulfoxide (DMSO) and tetrapropylammonium hydroxide (TPAOH) were employed as intercalants to delaminate $\mathrm{Ti}_{3} \mathrm{AlC}_{2}$ and $\mathrm{Nb}_{2} \mathrm{CT}_{x}$ MXenes, respectively ${ }^{25,26}$. With the help of sonication, thin layers of MXenes nanosheets that are electron transparent can be obtained (Fig. 1c, Supplementary Fig. 1d).

The chemical nature of the MXenes was investigated by X-ray absorption spectroscopy (XAS). Figure 1d shows that the Ti K edge X-ray absorption near edge spectroscopy (XANES) spectrum of $\mathrm{Ti}_{3} \mathrm{C}_{2} \mathrm{~T}_{x}$ has similar shape compared to that of bulk $\mathrm{TiC}$ rather than that of $\mathrm{Ti}$ foil or $\mathrm{TiO}_{2}$. The edge energy of $\mathrm{Ti}_{3} \mathrm{C}_{2} \mathrm{~T}_{x}$ $(4967.2 \mathrm{eV})$ is close to that of $\mathrm{TiC}(4967.1 \mathrm{eV})$, which is between the energies of $\mathrm{Ti}$ foil $(4966.4 \mathrm{eV})$ and $\mathrm{TiO}_{2}(4968.6 \mathrm{eV})$, indicating its carbide nature. The extended X-ray absorption fine structure (EXAFS) spectra (Fig. 1e) compare the local coordination of the Ti atoms in the $\mathrm{Ti}_{3} \mathrm{C}_{2} \mathrm{~T}_{x}$ MXene to its bulk counterpart (TiC). $\mathrm{Ti}_{3} \mathrm{C}_{2} \mathrm{~T}_{x}$ shows first-shell scattering $(\mathrm{Ti}-\mathrm{C} / \mathrm{O} / \mathrm{F})$ similar to that of $\mathrm{TiC}$ but with second-shell scattering (Ti-C-Ti) lower than that of $\mathrm{TiC}$, consistent with the reduced dimensionality of the $\mathrm{Ti}_{3} \mathrm{C}_{2} \mathrm{~T}_{x}$ MXene and corresponding high portion of surface $\mathrm{Ti}$ atoms. Similar results were obtained for $\mathrm{Nb}_{2} \mathrm{CT}_{x}$ by $\mathrm{Nb} \mathrm{K}$ edge XAS in our previous work ${ }^{16}$.

Characterizations of MXenes supported nanoparticles. Pt was loaded on the two MXene supports via incipient-wetness impregnation (IWI) as reported previously ${ }^{16}$. The Pt/MXene catalysts were further reduced with $5 \% \mathrm{H}_{2} / \mathrm{N}_{2}$ at $550{ }^{\circ} \mathrm{C}$, which is within the typical temperature range of LADH reactions ${ }^{27}$. High angle annular dark field scanning transmission electron microscopy (HAADF-STEM) shows that small NPs form on both catalysts. The average particle diameters are about $6 \pm 3.2 \mathrm{~nm}$ and $2.6 \pm 0.7 \mathrm{~nm}$ for $\mathrm{Pt} / \mathrm{Ti}_{3} \mathrm{C}_{2} \mathrm{~T}_{x}$ and $\mathrm{Pt} / \mathrm{Nb}_{2} \mathrm{CT}_{x}$ catalysts, respectively. The MXene supports enable dispersion of NPs without apparent agglomeration (Supplementary Figs. 2, 3).

The compositions of the NPs were first investigated by energy dispersive spectroscopy (EDS) elemental mapping. The signals of Pt and $\mathrm{Ti}$ or $\mathrm{Nb}$ overlap over all the NPs (Supplementary Figs. 4, 5), suggesting migrations of $M$ to the Pt NPs. Aberrationcorrected HAADF-STEM was utilized to further characterize the structures of Pt-Ti and Pt-Nb NPs. Figure 2a shows an image representative of the NPs supported by $\mathrm{Ti}_{3} \mathrm{C}_{2} \mathrm{~T}_{x}$ MXene. The NP in the center of the figure is viewed along the [111] zone axis, whereas another NP in the upper left corner is viewed along the [001] zone axis. Two different types of atoms can be clearly differentiated due to the Z-contrast in high angle annular dark field (HAADF) imaging. The bright dots are characteristic of heavier Pt atoms, while the dimmer ones correspond to Ti. Along 
a
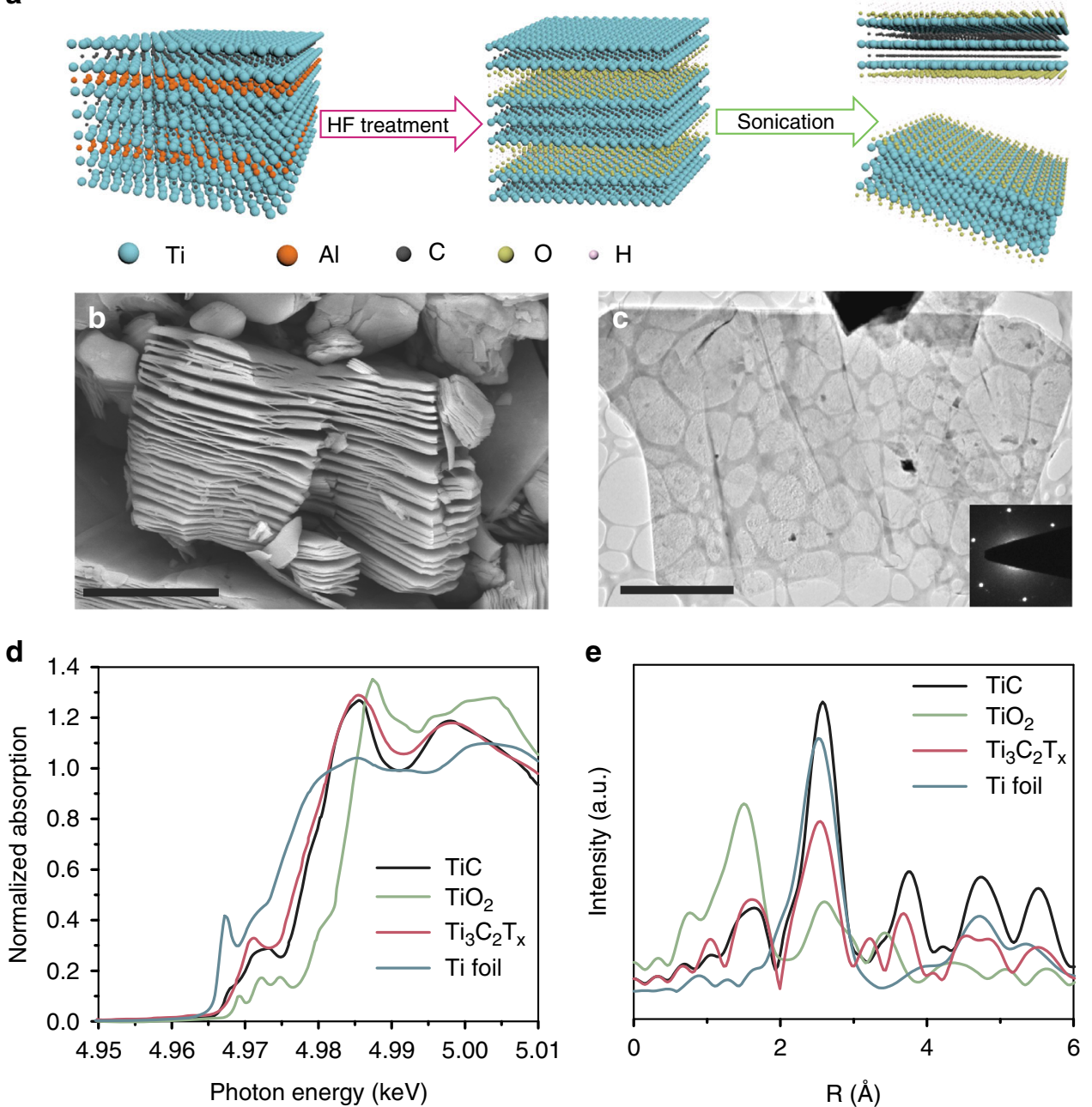

Fig. 1 Characterization of $\mathrm{Ti}_{3} \mathrm{C}_{2} \mathrm{~T}_{x} M$ Xene support. a Schematic of $\mathrm{Ti}_{3} \mathrm{C}_{2} \mathrm{~T}_{x} M$ Xene preparation. $\mathbf{b} \mathrm{SEM}$ image of $\mathrm{Ti}_{3} \mathrm{C}_{2} \mathrm{~T}_{x} M X$ Xene, the scale bar corresponds to $3 \mu \mathrm{m}$. c TEM image of $\mathrm{Ti}_{3} \mathrm{C}_{2} \mathrm{~T}_{x} \mathrm{MXene}$ nanosheets. Inset represents the selected area electron diffraction (SAED) pattern showing hexagonal basal plane symmetry of $\mathrm{Ti}_{3} \mathrm{C}_{2} \mathrm{~T}_{x} \mathrm{MXene}$. The scale bar corresponds to $2 \mu \mathrm{m}$. $\mathbf{d}$ XANES spectra of the $\mathrm{Ti}_{3} \mathrm{C}_{2} \mathrm{~T}_{x}$ compared to references including Ti metal foil, $\mathrm{TiO}_{2}$ and TiC. e Magnitude of the Fourier transform of the $\mathrm{k}^{2}$ weighted EXAFS of the $\mathrm{Ti}_{3} \mathrm{C}_{2} \mathrm{~T}_{x}$ compared to bulk TiC

the [111] axis of a NP, the projected unit cells are composed of periodic hexagonal arrays of $\mathrm{Pt}$ atoms that surround $\mathrm{Ti}$ atoms at the center of the hexagons (Fig. 2b), which indicates a specific $\mathrm{L}_{2}$ type symmetry and is consistent with formation of the $\mathrm{Cu}_{3} \mathrm{Au}$ type $\mathrm{Pt}_{3} \mathrm{Ti}$ IMC (Fig. $\left.2 \mathrm{c}\right)^{22}$. The experimental HAADF-STEM image shows good agreement with the simulated (111) surface of $\mathrm{L}_{2}$ ordered $\mathrm{Pt}_{3} \mathrm{Ti}$ nanostructures (inset of Fig. $2 \mathrm{~b}$ ). The $\mathrm{Pt} / \mathrm{Ti}$ ratio is estimated by EDS elemental mapping on a NP hanging over the vacuum to avoid the Ti signals from the $\mathrm{Ti}_{3} \mathrm{C}_{2} \mathrm{~T}_{x}$ MXene support and give a value of the molar ratio of $\mathrm{Pt}$ to Ti equal to 3.55 (Supplementary Fig. 6), which is close to the theoretical ratio of $\mathrm{Pt}_{3} \mathrm{Ti}$ alloy within error.

The ordered intermetallic structure of Pt-Nb NPs can also be directly observed (circled with dash lines) despite the small particle sizes $(2.6 \pm 0.7 \mathrm{~nm})$ (Fig. 2d). The fcc structure is identified by two NPs viewed along [111] and [001] axis, respectively (Fig. 2e, Supplementary Fig. 7). The inset of Fig. 2e shows the fast Fourier transform (FFT) pattern of the NP viewed

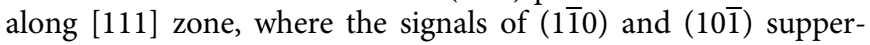
lattice are present. Since the unique super periods are present only in the structurally ordered intermetallic phase and are absent in the disordered alloy phase, ordering in the NP is confirmed. Moreover, the enlarged image of the NP (inset of Fig. 2f) shows a dimmer $\mathrm{Nb}$ atom surrounded by six Pt atoms in a hexagonal pattern, again implying a local $\mathrm{Ll}_{2}$ ordering. The intermetallic phase, however, does not form over the whole NP. The distribution of the Pt-Nb intermetallic order in the NP is demonstrated by the contrast variation of the inverse fast Fourier transform (IFFT) image in Fig. 2f. The IFFT pattern shows that the ordered Pt-Nb phase preferentially populates the edge of the $\mathrm{NP}$ versus the inner core. This is consistent with previous studies reporting that the diffusion of the second metallic species into the noble metal lattice (from the NP surface) played a pivotal role in the formations of intermetallic $\mathrm{NPs}^{28-30}$.

To investigate the altered Pt chemical environment resulting from the RMSI between Pt and MXenes, the Pt/MXene catalysts were studied by in situ XAS. A Pt/SiO 2 catalyst with an average particle size of $1.8 \pm 0.6 \mathrm{~nm}$ was also prepared as a reference (Supplementary Fig. 8). The EXAFS spectra of both in situ reduced catalysts (Fig. 3a) show metal-metal scattering significantly different from the typical three-peak pattern characteristic of monometallic Pt NPs on $\mathrm{SiO}_{2}$. For $\mathrm{Pt} / \mathrm{Ti}_{3} \mathrm{C}_{2} \mathrm{~T}_{x}$, the central peak of the magnitude of Fourier transform EXAFS has a higher intensity due to the in-phase constructive interference between $\mathrm{Pt}-\mathrm{Ti}$ and Pt-Pt scattering, while the opposite is observed on $\mathrm{Pt} / \mathrm{Nb}_{2} \mathrm{CT}_{x}$ because $\mathrm{Pt}-\mathrm{Nb}$ and $\mathrm{Pt}-\mathrm{Pt}$ are out-of-phase. 
a

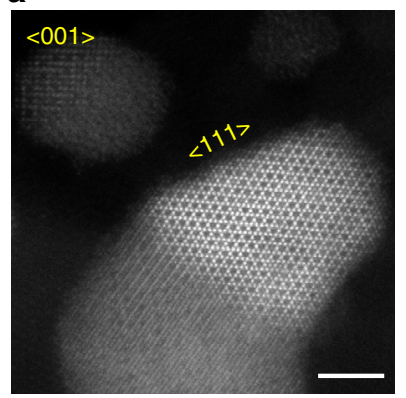

d

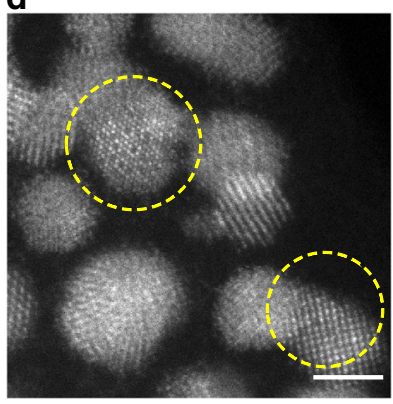

b

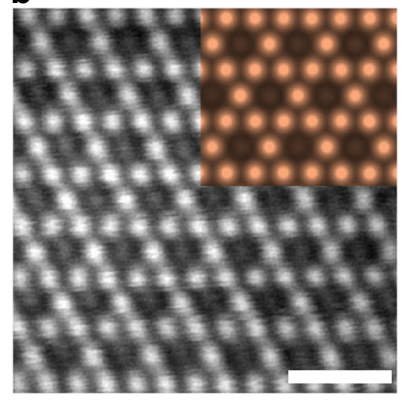

e

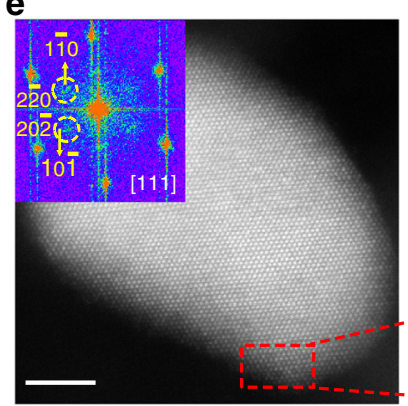

C

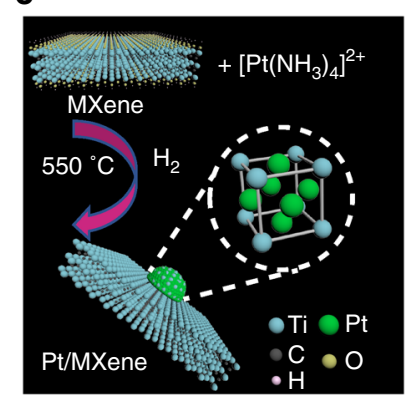

f

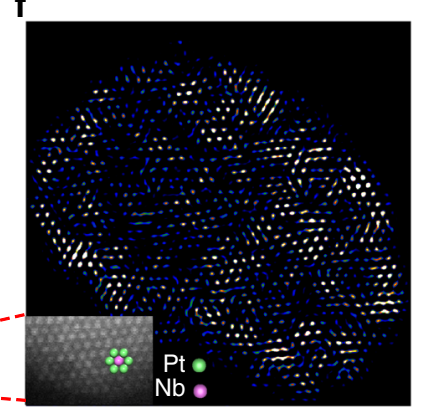

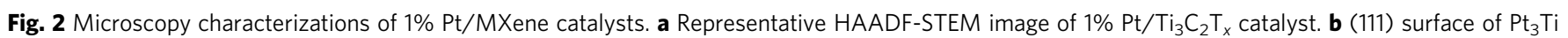

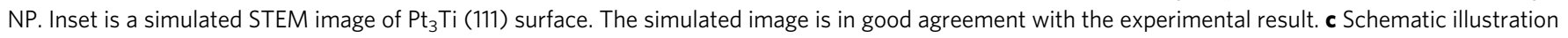

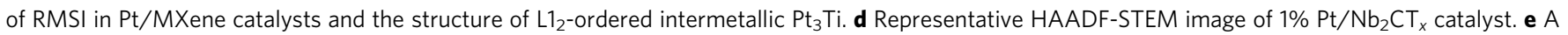

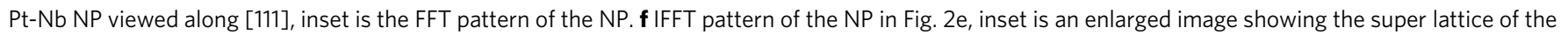
NP. Scale bars: a, d, e $2 \mathrm{~nm}$, and b $500 \mathrm{pm}$

Quantitative fitting (Supplementary Fig. 9, Supplementary Table 1) of the EXAFS gives the following average coordination numbers (CNs) and bond distances: 6.6 Pt-Pt bond and 3.4 Pt-Ti bond both at $2.75 \AA$ for the $\mathrm{Pt} / \mathrm{Ti}_{3} \mathrm{C}_{2} \mathrm{~T}_{x}$ catalyst and $6.7 \mathrm{Pt}-\mathrm{Pt}$ bond at $2.77 \AA$ and $1.8 \mathrm{Pt}-\mathrm{Nb}$ bond at $2.75 \AA$ for the $\mathrm{Pt} / \mathrm{Nb}_{2} \mathrm{CT}_{x}$ catalyst. The EXAFS of both catalysts confirms the presence of bimetallic NPs. For an ideal bulk $\mathrm{L}_{2}$ type $\mathrm{Pt}_{3} \mathrm{M}$ intermetallic structure, the bond distances are the same for the Pt-Pt and Pt-M paths and the ratio of $\mathrm{Pt}-\mathrm{Pt} \mathrm{CN}$ over Pt-M CN is 2. Thus, the XAS results for $\mathrm{Pt} / \mathrm{Ti}_{3} \mathrm{C}_{2} \mathrm{~T}_{x}$ indicate the formation of $\mathrm{Ll}_{2}$ type $\mathrm{Pt}_{3} \mathrm{M}$ structures, which is concordant with those of STEM. For the Pt/ $\mathrm{Nb}_{2} \mathrm{CT}_{x}$, the Pt-Pt/Pt-Nb CN ratio is much greater than 2 and their bond distances are slightly different, consistent with formation of partial/surface $\mathrm{Ll}_{2}$ ordering in the Pt-Nb NPs as observed by STEM. Together the HAADF-STEM and EXAFS indicate that RMSI occurs in these two MXene supported catalysts, leading to formations of IMCs with tunable compositions and structures. We note for comparison that $\mathrm{Pt}$ added similarly to bulk titanium carbide or niobium carbide surfaces did not produce IMCs (Supplementary Figs. 10, 11).

Electronic structures of Pt/MXene catalysts. In situ XANES at Pt $\mathrm{L}_{\mathrm{III}}$ edge was conducted on the reduced catalysts to examine the energy of the unoccupied $d$ states (Fig. 3b). The XANES energies of the $\mathrm{Pt} / \mathrm{Ti}_{3} \mathrm{C}_{2} \mathrm{~T}_{x}$ and $\mathrm{Pt} / \mathrm{Nb}_{2} \mathrm{CT}_{x}$ catalysts are 11564.6 $\mathrm{eV}$ and $11564.3 \mathrm{eV}$, respectively, both higher compared to that of monometallic Pt $(11564.0 \mathrm{eV})$. The whitelines are slightly taller and narrower compared to that of $\mathrm{Pt} / \mathrm{SiO}_{2}$, corresponding to a change in the energy distribution of the $5 d$ unoccupied states. Shifts in electronic band energy level were also detected by XPS (in situ reduced samples). In Fig. $3 c$, the Pt $4 f_{7 / 2}$ binding energies of $\mathrm{Pt} / \mathrm{Ti}_{3} \mathrm{C}_{2} \mathrm{~T}_{x}$ and $\mathrm{Pt} / \mathrm{Nb}_{2} \mathrm{CT}_{x}$ are $71.3 \mathrm{eV}$ and $71.6 \mathrm{eV}$ respectively, which shift to higher values with respect to that of monometallic Pt $(70.8 \mathrm{eV})$. These spectroscopic studies of fully reduced samples indicate that the formation of $\mathrm{Pt}_{3} \mathrm{M}$ intermetallic
NPs leads to altered electronic structure of the Pt atoms and that the $5 d$ states are shifted to higher energy in $\mathrm{Pt} / \mathrm{MXenes}$ compared to $\mathrm{Pt} / \mathrm{SiO}_{2}$.

To better understand the modulated electronic structures of the $\mathrm{Pt} / \mathrm{MX}$ ene catalysts owing to the formations of IMCs, we carried out density functional theory (DFT) calculations of the projected density of states (DOS). Out of the two Pt/MXene catalysts, the $\mathrm{Pt} / \mathrm{Ti}_{3} \mathrm{C}_{2} \mathrm{~T}_{x}$ catalyst contains full (instead of partial) IMC NPs with the exposure of representative (111) surface planes resolved by aberration-corrected STEM. Therefore, we employed slab models of $\mathrm{Pt}_{3} \mathrm{Ti}(111)$ and $\mathrm{Pt}(111)$ surfaces for comparison. As shown in Fig. 3d, the $d$ band center of $\mathrm{Pt}(111)$ is located at $-1.97 \mathrm{eV}$ relative to the Fermi level, whereas that of $\mathrm{Pt}_{3} \mathrm{Ti}(111)$ downshifts to $-2.37 \mathrm{eV}$ due to a strong Pt-Ti $d$ - $d$ orbital coupling. Similar shifts of $d$ band center to a lower level were also reported in other Pt-M systems, where $\mathrm{M}$ is $\mathrm{Co}, \mathrm{Fe}, \mathrm{Ni}, \mathrm{Ce}^{31}$. Additionally, $\sim 1 \mathrm{eV}$ above the Fermi level, the DOS calculation also shows an increased intensity for $\mathrm{Pt}_{3} \mathrm{Ti}(111)$ (Fig. 3d) compared to $\mathrm{Pt}(111)$, which is consistent with the in situ XANES results showing higher energy and intensity of the whiteline of the $\mathrm{Pt} / \mathrm{Ti}_{3} \mathrm{C}_{2} \mathrm{~T}_{x}$ catalyst compared to $\mathrm{Pt} / \mathrm{SiO}_{2}$. A recent study combining DFT calculations and an emerging technique, in situ resonant inelastic X-ray scattering (RIXS), reported that formation of IMCs leads to a downward shift of the $d$ band center as well as an upward shift of the energy of the unoccupied $5 d$ states $^{32}$, which agrees with our results.

Catalytic performance and free energy calculations. According to $d$ band chemisorption theory, shifts to lower energy of $d$ band center will reduce the surface adsorption reactivity ${ }^{33}$ and directly affect the chemistry of catalysts ${ }^{34}$. LADH reactions are sensitive to the energy level of $d$ band electrons of the catalysts surface ${ }^{21}$, thus were used as probes to evaluate effects of the in situ formation of $\mathrm{Pt}_{3} \mathrm{M}$ intermetallic NPs on the catalytic performance. All the catalysts were pretreated and tested under the same 
a

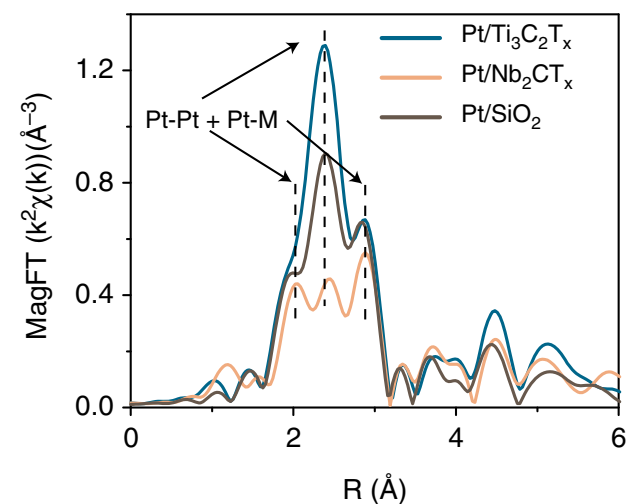

C

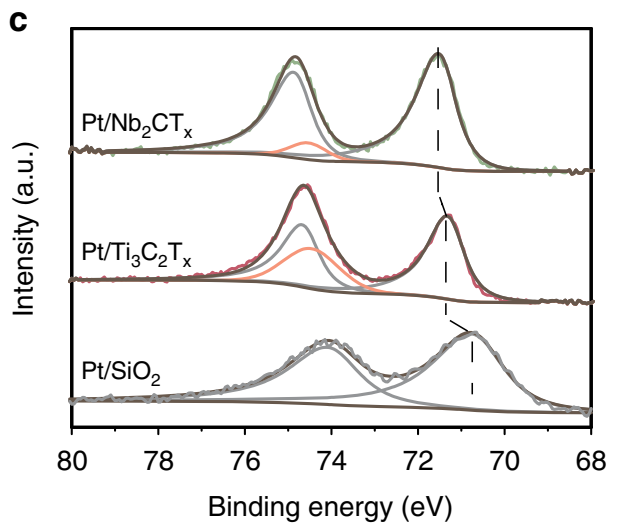

b

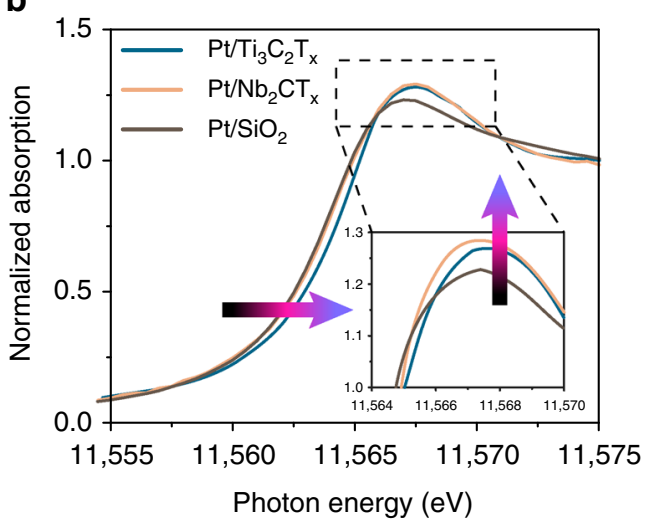

d

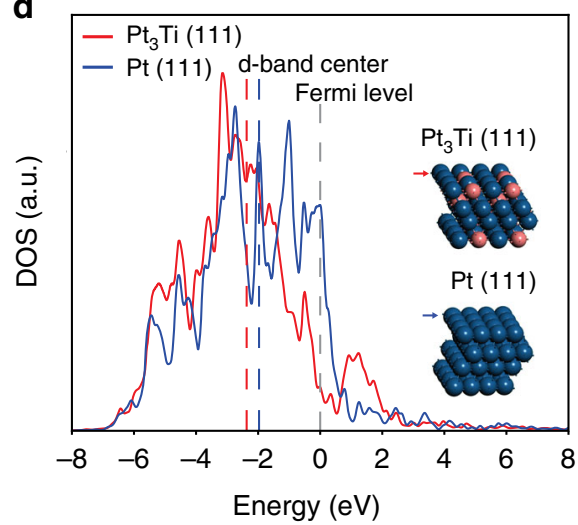

Fig. 3 In situ Spectroscopy characterization of Pt/MXenes catalysts. a Magnitude of the Fourier Transform of the $k^{2}$ weighted Pt $\mathrm{L}_{\text {III }}$ edge in situ EXAFS of the $\mathrm{Pt} / \mathrm{Ti}_{3} \mathrm{C}_{2} \mathrm{~T}_{x}$ and $\mathrm{Pt} / \mathrm{Nb}_{2} \mathrm{CT}_{x}$ catalyst after reduction at $550^{\circ} \mathrm{C}$ in $\mathrm{H}_{2}$ compared to $\mathrm{Pt} / \mathrm{SiO}_{2}$. $\mathbf{b}$ The $\mathrm{Pt} \mathrm{L}_{\| 1 \mid}$ edge in situ XANES spectra of the Pt/Ti ${ }_{3} \mathrm{C}_{2} \mathrm{~T}_{x}$ and $\mathrm{Pt} / \mathrm{Nb}_{2} \mathrm{CT}_{x}$ catalyst after reduction at $550{ }^{\circ} \mathrm{C}$ in $\mathrm{H}_{2}$ compared to $\mathrm{Pt} / \mathrm{SiO}_{2}$. c XPS spectra of $\mathrm{Pt} / \mathrm{Ti}_{3} \mathrm{C}_{2} \mathrm{~T}_{x}, \mathrm{Pt} / \mathrm{Nb}_{2} \mathrm{CT}_{x}$ and $\mathrm{Pt} / \mathrm{SiO} \mathrm{O}_{2}$ reduced at $550{ }^{\circ} \mathrm{C}$ by $\mathrm{H}_{2}$ in a spectrometer side chamber and not exposed to air. $\mathbf{d}$ DFT calculated projected density of states (DOS) for the $5 d$ orbitals of $\mathrm{Pt}_{\mathrm{t}}$ in the top-layer $\mathrm{Pt}{ }_{3} \mathrm{Ti}$ (111) and Pt (111)

conditions summarized in Methods. The product selectivity of different catalysts was compared between 0 and 20\% light alkane conversions. For both dehydrogenation of propane (Fig. 4a) and isobutane (Supplementary Fig. 12), Pt/MXene catalysts are much more selective than $\mathrm{Pt} / \mathrm{SiO}_{2}$ at the same conversion. For example, when the conversion of propane is $15 \%, \mathrm{Pt} / \mathrm{SiO}_{2}$ is $60 \%$ selective to propylene, while those of $\mathrm{Pt} / \mathrm{Ti}_{3} \mathrm{C}_{2} \mathrm{~T}_{x}$ and $\mathrm{Pt} / \mathrm{Nb}_{2} \mathrm{CT}_{x}$ are $\sim 95 \%$ and $\sim 90 \%$, respectively (Fig. $4 \mathrm{a}$ ). Though intermetallic catalysts have larger particle size compared to the reference monometallic Pt catalyst, the improvement in their catalytic performance can be attributed to the formation of intermetallic structure rather than a size effect since larger particles have been reported to give lower selectivity 35,36 . For all the catalysts, the selectivity of dehydrogenation is lower at higher conversion due to the hydrogenolysis side reaction that requires hydrogen. The decrease in selectivity under increasing conversion is reduced on the $\mathrm{Pt} /$ $\mathrm{Ti}_{3} \mathrm{C}_{2} \mathrm{~T}_{x}$ and $\mathrm{Pt} / \mathrm{Nb}_{2} \mathrm{CT}_{x}$ catalysts, indicating that the effect of side reactions is less prominent on the intermetallic NPs. The TORs were calculated using the reaction rate per gram of Pt measured under differential conditions and catalyst dispersion estimated from the average particles sizes. For propane dehydrogenation $(\mathrm{PDH})$, the TORs were $0.12 \mathrm{~s}^{-1}, 0.09 \mathrm{~s}^{-1}$ and $0.08 \mathrm{~s}^{-1}$ for Pt/ $\mathrm{SiO}_{2}, \mathrm{Pt} / \mathrm{Nb}_{2} \mathrm{CT}_{x}$ and $\mathrm{Pt} / \mathrm{Ti}_{3} \mathrm{C}_{2} \mathrm{~T}_{x}$, respectively. The TORs for isobutane dehydrogenation followed a similar trend and were $0.09 \mathrm{~s}^{-1}, 0.06 \mathrm{~s}^{-1}$ and $0.06 \mathrm{~s}^{-1}$ for $\mathrm{Pt} / \mathrm{SiO}_{2}, \mathrm{Pt} / \mathrm{Nb}_{2} \mathrm{CT}_{x}$ and $\mathrm{Pt} /$ $\mathrm{Ti}_{3} \mathrm{C}_{2} \mathrm{~T}_{x}$. These values are similar to the TORs reported for typical LADH catalysts ${ }^{21}$. The evolution of performance with time onstream for IMC catalysts are also consistent with the monometallic Pt catalyst (Supplementary Fig. 13) as well as previous literature, due to slow deposition of coke ${ }^{21}$. The used $\mathrm{Pt} /$ $\mathrm{Nb}_{2} \mathrm{CT}_{x}$ and $\mathrm{Pt} / \mathrm{Ti}_{3} \mathrm{C}_{2} \mathrm{~T}_{x}$ were further characterized by HAADFSTEM to check the stability of the IMCs. The structures of $\mathrm{Pt}_{3} \mathrm{Ti}$ and $\mathrm{Pt}_{3} \mathrm{Nb}$ are preserved in the spent catalysts (Supplementary Figs. 14, 15), indicating that the IMCs NPs were stable under the LADH reaction conditions.

To understand the high olefin selectivity of the intermetallic catalysts for $\mathrm{PDH}$ reaction, energy profiles of $\mathrm{PDH}$ reaction and possible side reactions were studied by DFT calculations. Snapshots of structures of reaction intermediates and transition states were illustrated in Fig. 4b, with the free energies of the relevant reaction pathways on $\mathrm{Pt}(111)$ and $\mathrm{Pt}_{3} \mathrm{Ti}(111)$ surfaces calculated and shown in Fig. 4c. PDH follows a step-wise $\mathrm{C}-\mathrm{H}$ bond breaking process 37 , which starts with dissociative adsorption of propane forming surface alkyl species (intermediate 2), followed by the scission of a secondary $\mathrm{C}-\mathrm{H}$ bond generating adsorbed olefins (intermediate 4). Our DFT results show that the free energy changes and barriers of the first two steps on the $\mathrm{Pt}_{3} \mathrm{Ti}(111)$ are higher than those on pure $\mathrm{Pt}(111)$, indicating weakened surface adsorption activity of the intermetallic phase consistent with the shifts in the $5 d$ DOS indicated by DFT, XANES, and XPS. In the following steps, the adsorbed olefins may undergo desorption, further (deep) dehydrogenation (intermediate 7 ) or $\mathrm{C}-\mathrm{C}$ breaking (intermediate 11) processes. The latter two steps are believed to generate the precursors leading to side reactions, i.e., coking and hydrogenolysis ${ }^{38}$. 
a

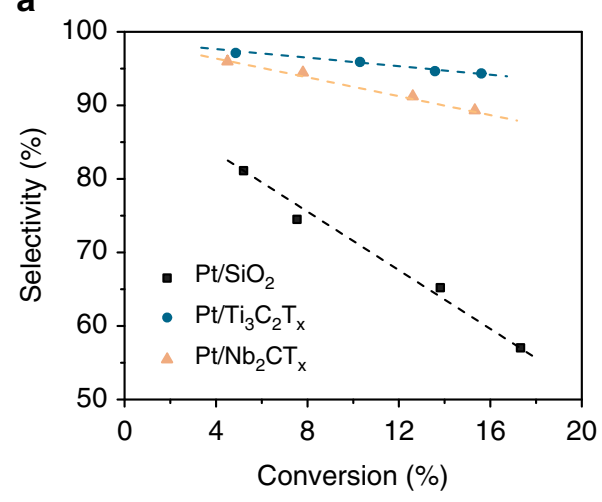

b
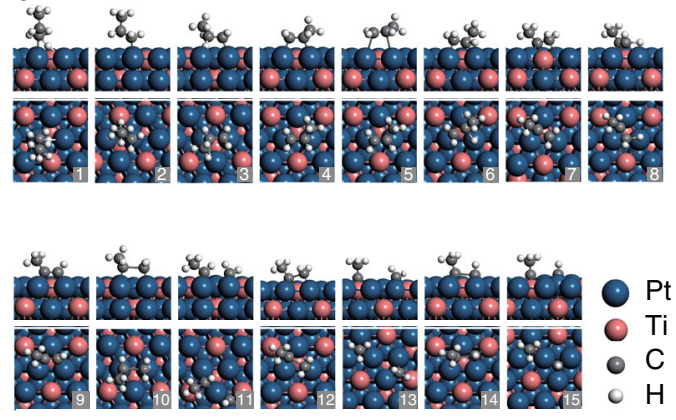

C

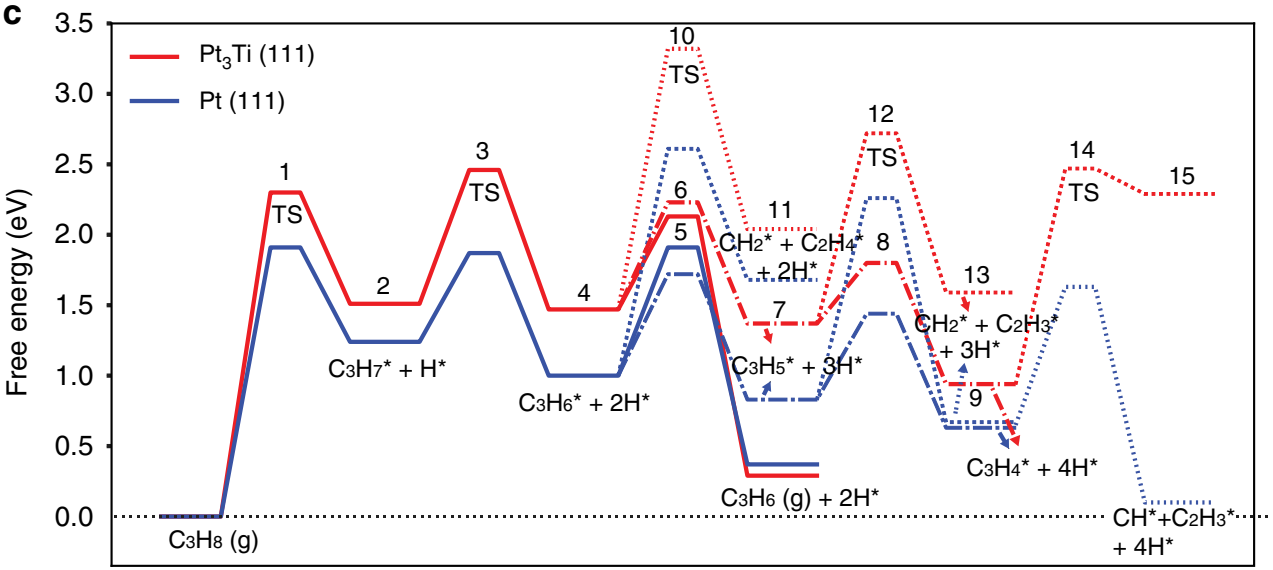

Reaction coordinate

Fig. 4 Catalytic performance and DFT calculation of Pt/MXene catalysts. a Plots of conversion vs. selectivity for propane dehydrogenation measured in $200 \mathrm{~cm}^{3} \mathrm{~min}^{-1}$ of $2.5 \% \mathrm{C}_{3} \mathrm{H}_{8}, 2.5 \% \mathrm{H}_{2}$ balanced in $\mathrm{N}_{2}$ at $1.5 \mathrm{~atm}$ and $550{ }^{\circ} \mathrm{C}$ for $\mathrm{Pt} / \mathrm{Ti}_{3} \mathrm{C}_{2} \mathrm{~T}_{x_{1}} \mathrm{Pt} / \mathrm{Nb}_{2} \mathrm{CT}_{x}$, and Pt/SiO $\mathrm{S}_{2}$ catalyst. b Snapshots of optimized structures as numbered in $\mathbf{c}$ from side and top view angles $\left(\mathrm{H}^{*}\right.$ is not shown). c DFT-calculated free energy diagram of relevant (side-)reaction steps in propane dehydrogenation on $\mathrm{Pt}_{3} \mathrm{Ti}$ (111) and $\mathrm{Pt}$ (111) surfaces. The dotted lines denote the $\mathrm{C}-\mathrm{C}$ cracking reactions of $\mathrm{C}_{3} \mathrm{H}_{6}{ }^{\star}, \mathrm{C}_{3} \mathrm{H}_{5}{ }^{\star}$, and $\mathrm{C}_{3} \mathrm{H}_{4}{ }^{\star}$, generating $\mathrm{CH}_{2}{ }^{\star}+\mathrm{C}_{2} \mathrm{H}_{4}{ }^{\star}, \mathrm{CH}_{2}{ }^{\star}+\mathrm{C}_{2} \mathrm{H}_{3}{ }^{\star}$, and $\mathrm{CH}^{\star}+\mathrm{C}_{2} \mathrm{H}_{3}{ }^{\star}$, respectively. The dash-dot lines denote the further dehydrogenation of $\mathrm{C}_{3} \mathrm{H}_{6}{ }^{\star}$ to $\mathrm{C}_{3} \mathrm{H}_{5}{ }^{\star}$, and $\mathrm{C}_{3} \mathrm{H}_{5}{ }^{\star}$ to $\mathrm{C}_{3} \mathrm{H}_{4}^{*}$

On $\mathrm{Pt}(111)$, the free energy barrier for dehydrogenation of $\mathrm{C}_{3} \mathrm{H}_{6}{ }^{*}$ to $\mathrm{C}_{3} \mathrm{H}_{5}{ }^{*}$ is $0.19 \mathrm{eV}$ lower than that of propylene desorption, indicating that deep dehydrogenation is more favorable on $\mathrm{Pt}(111)$ surface. In contrast, on $\mathrm{Pt}_{3} \operatorname{Ti}(111)$ the $\mathrm{C}_{3} \mathrm{H}_{6}{ }^{*}$ desorption is more favored by $0.1 \mathrm{eV}$ in barrier than further dehydrogenation. For direct comparison, the barrier of $\mathrm{C}_{3} \mathrm{H}_{6}{ }^{*}$ desorption on $\mathrm{Pt}_{3} \mathrm{Ti}(111)(0.66 \mathrm{eV})$ is $0.25 \mathrm{eV}$ lower than that on $\mathrm{Pt}(111)(0.91 \mathrm{eV})$. In addition, the free energy change of $\mathrm{C}_{3} \mathrm{H}_{6}{ }^{*}$ desorption on $\mathrm{Pt}_{3} \mathrm{Ti}(111)(-1.17 \mathrm{eV})$ is $0.54 \mathrm{eV}$ more favorable than that on $\mathrm{Pt}(111)(-0.63 \mathrm{eV})$. Overall, the introduction of Ti lowers the desorption barrier of $\mathrm{C}_{3} \mathrm{H}_{6}{ }^{*}$ to the gas phase $\left(0.66 \mathrm{eV}\right.$ of $\mathrm{Pt}_{3} \mathrm{Ti}(111)$ vs. $0.91 \mathrm{eV}$ of $\left.\mathrm{Pt}(111)\right)$ and increases the energy barrier for deep dehydrogenation. On both surfaces, $\mathrm{C}-\mathrm{C}$ cracking of dehydrogenated reaction intermediates, e.g., $\mathrm{C}_{3} \mathrm{H}_{6}{ }^{*}, \mathrm{C}_{3} \mathrm{H}_{5}{ }^{*}$, and $\mathrm{C}_{3} \mathrm{H}_{4}{ }^{*}$, requires much higher activation energies and thus are less competitive. Nevertheless, on $\mathrm{Pt}_{3} \mathrm{Ti}$ (111) the $\mathrm{C}-\mathrm{C}$ cracking steps (intermediates 11,13 , and 15) are all endergonic and hence are hindered compared with $\mathrm{Pt}(111)$ where $\mathrm{C}_{3} \mathrm{H}_{5}{ }^{*}$ and $\mathrm{C}_{3} \mathrm{H}_{4}{ }^{*}$ cracking are exergonic and much more favorable. These results rationalize the observed higher selectivity toward propylene for $\mathrm{PDH}$ by $\mathrm{Pt}_{3} \mathrm{Ti}$ intermetallic NPs compared to pure Pt.

Our experimental results and DFT calculations show that the $\mathrm{Pt}_{3} \mathrm{Ti}$ intermetallic phase has a lower $d$ band center compared with that of monometallic Pt, which results in weaker adsorption of light hydrocarbon species and changes of the relative free energy and barriers of the reaction steps during dehydrogenation and side reactions. Lowering of the olefin desorption barrier to below that of deep dehydrogenation and $\mathrm{C}-\mathrm{C}$ breaking contributes to the high catalyst selectivity. The same type of calculations were not conducted for the $\mathrm{Pt} / \mathrm{Nb}_{2} \mathrm{CT}_{x}$ catalyst due to its relatively less well-defined structure. However, similar catalyst electronic structure compared to $\mathrm{Pt} / \mathrm{Ti}_{3} \mathrm{C}_{2} \mathrm{~T}_{x}$ can be expected according to the in situ X-ray spectroscopy results. The adsorption properties, and reaction energetics are, therefore, also expected to be similar. The fact that the $\mathrm{Pt} / \mathrm{Nb}_{2} \mathrm{CT}_{x}$ catalyst has a slightly lower selectivity compared to $\mathrm{Pt} / \mathrm{Ti}_{3} \mathrm{C}_{2} \mathrm{~T}_{x}$ is likely due to the extent of the IMCs formation, i.e., full verses surface IMCs, and differences in electronic effects. These additional subtle catalytic differences demonstrate that the chemical properties of the catalysts are tunable using different MXene materials as the RMSI-active supports.

In summary, this work demonstrates two IMC catalysts selective for LADH achieved by RMSI between Pt NPs and $\mathrm{Ti}_{3} \mathrm{C}_{2} \mathrm{~T}_{x}$ and $\mathrm{Nb}_{2} \mathrm{CT}_{x}$ MXenes. The intermetallic surface is imaged by atomic resolution HAADF-STEM and its high catalytic selectivity is rationalized by DFT calculations. With MXenes as catalyst supports and through their active interactions 
with metal NPs, there is an opportunity to explore many new compositions for heterogeneous catalysis in industrial gas-phase reactions as well as electrochemical conversion, with the possibility that the chemical and electronic properties of the resulting catalysts can be tuned over a wider range than what is currently possible using conventional catalyst supports.

\section{Methods}

Synthesis of $\mathrm{Ti}_{3} \mathbf{A l C}_{\mathbf{2}}$ and $\mathbf{N b}_{\mathbf{2}} \mathbf{A I C}$. The $\mathrm{Ti}_{3} \mathrm{AlC}_{2}$ powder was synthesized by spark plasma sintering (SPS) of $\mathrm{TiH}_{2} / \mathrm{Al} / \mathrm{TiC}$. Commercial powders of titanium (II) hydride, aluminum and titanium (IV) carbide were mixed in a molar ratio of $\mathrm{TiH}_{2} /$ $\mathrm{Al} / \mathrm{TiC}=1: 1: 1.8$ in a graphite die coated with boron nitride (BN). Excess $\mathrm{Al}$ and less than a full equivalent of $\mathrm{TiC}$ were added because a small portion of $\mathrm{Al}$ will be lost during high-temperature processing, and carbon deficiencies exist in most $\mathrm{Al}$ containing MAX phases. Then, the material was loaded in a Fuji-211lx SPS system and sintered at $1350{ }^{\circ} \mathrm{C}$ under $30 \mathrm{MPa}$ for $1 \mathrm{~h}$. The resulting $\mathrm{Ti}_{3} \mathrm{AlC}_{2}$ was then pulverized and sieved through a 325-mesh screen.

$\mathrm{The} \mathrm{Nb}_{2} \mathrm{AlC}$ powder was synthesized by SPS of $\mathrm{Nb} / \mathrm{Al} /$ graphite mixtures. Commercial powders of niobium, graphite and aluminum were mixed in a molar ratio of Nb:Al:C = 2:1.4:0.9 in a graphite die coated with boron nitride (BN). Then, the material was loaded in a Fuji-211lx SPS system and sintered at $1500^{\circ} \mathrm{C}$ under $30 \mathrm{MPa}$ for $1 \mathrm{~h}$. The resulting $\mathrm{Nb}_{2} \mathrm{AlC}$ was then pulverized and sieved through a 400-mesh screen.

Synthesis of $\mathbf{T i}_{\mathbf{3}} \mathbf{C}_{\mathbf{2}} \mathbf{T}_{\boldsymbol{x}}$ and $\mathbf{N b}_{\mathbf{2}} \mathbf{C T}$. Preparation of $\mathrm{Ti}_{3} \mathrm{C}_{2} \mathrm{~T}_{x}$ MXene: Approximately $1 \mathrm{~g}$ of the prepared $\mathrm{Ti}_{3} \mathrm{AlC}_{2}$ powder was immersed in $10 \mathrm{~mL}$ of $50 \%$ aqueous hydrofluoric acid solution stirred with a magnetic bar for approximately 1 days at $35^{\circ} \mathrm{C}$. The resulting MXene suspension was repeatedly washed with deionized water (DI) and centrifuged until the $\mathrm{pH}$ reached $\sim 5$. The final MXene was dried under vacuum at room temperature and stored in a glove box until usage.

Preparation of $\mathrm{Nb}_{2} \mathrm{CT}_{x}$ MXene: Approximately $1 \mathrm{~g}$ of the prepared $\mathrm{Nb}_{2} \mathrm{AlC}$ powder was immersed in $10 \mathrm{~mL}$ of $50 \%$ aqueous hydrofluoric acid (HF) solution stirred with a magnetic bar for approximately 3 days at $55^{\circ} \mathrm{C}$. The resulting MXene suspension was repeatedly washed with deionized water (DI) and centrifuged until the $\mathrm{pH}$ reached $\sim 5$. The final MXene was dried under vacuum at room temperature and stored in a glove box until usage.

Catalyst preparation. A monometallic Pt catalyst (2 wt. \% Pt supported on Davisil 636 silica gel from Sigma-Aldrich) was synthesized using the incipient wetness impregnation (IWI) method. $0.20 \mathrm{~g}$ of tetraammineplatinum nitrate was dissolved in $3 \mathrm{~mL}$ of $\mathrm{H}_{2} \mathrm{O}$.Approximately $30 \%$ ammonium hydroxide solution $\left(\mathrm{NH}_{4} \mathrm{OH}, 28 \%\right.$ $\mathrm{NH}_{3}$ in $\mathrm{H}_{2} \mathrm{O}, \geq 99.99 \%$, Sigma-Aldrich) was then added to the solution until the $\mathrm{pH}$ reached 11. The obtained Pt precursor solution was added dropwise to $5 \mathrm{~g}$ of silica and stirred. After drying overnight under vacuum, the sample was calcined at $225^{\circ}$ $\mathrm{C}$ for $3 \mathrm{~h}$ and reduced at $550{ }^{\circ} \mathrm{C}$ in $5 \% \mathrm{H}_{2} / \mathrm{N}_{2}$ for $30 \mathrm{~min}$.

Pt supported on $\mathrm{Ti}_{3} \mathrm{C}_{2} \mathrm{~T}_{x}$ and on $\mathrm{Nb}_{2} \mathrm{CT}_{x}$ were prepared via a similar method. $0.20 \mathrm{~g}$ of tetraammineplatinum nitrate $\mathrm{Pt}\left(\mathrm{NH}_{3}\right)_{4}\left(\mathrm{NO}_{3}\right)_{2}$ were dissolved in $0.5 \mathrm{~mL}$ of $\mathrm{H}_{2} \mathrm{O}$ to prepare $1 \mathrm{~mol} \mathrm{~L}^{-1} \mathrm{Pt}$ precursor solution. $0.05 \mathrm{~mL}$ of such solution was impregnated on fresh $\mathrm{Ti}_{3} \mathrm{C}_{2} \mathrm{~T}_{x}$ and $\mathrm{Nb}_{2} \mathrm{CT}_{x}$, respectively, prior to dying overnight under vacuum. The obtained catalysts were reduced at $550{ }^{\circ} \mathrm{C}$ in $5 \% \mathrm{H}_{2} / \mathrm{N}_{2}$ for at least $0.5 \mathrm{~h}$ before each catalytic test or characterization.

LADH kinetics. LADH kinetics measurements were carried out in a quartz fixedbed reactor with $3 / 8$-inch ID. Catalysts around $0.02-0.15 \mathrm{~g}$ were diluted using pure $\mathrm{SiO}_{2}$ to achieve a total weight of $1.00 \mathrm{~g}$ for testing the performance. Reaction temperature was measured using a thermocouple inserted in a stainless-steel thermocouple well locating at the bottom center of the catalyst bed. Agilent 7890A gas chromatograph system quipped with a flame ionization detector (FID) was employed for analyzing the products. Prior to each measurement, the fresh catalysts were reduced by $5 \% \mathrm{H}_{2} / \mathrm{N}_{2}\left(50 \mathrm{~cm}^{3} \mathrm{~min}^{-1}\right)$ for $30 \mathrm{~min}$ at $550{ }^{\circ} \mathrm{C}$ with the temperature ramping rate of $15^{\circ} \mathrm{C} \mathrm{min}-1$. Propane dehydrogenation was tested under a reaction atmosphere of $2.5 \% \mathrm{C}_{3} \mathrm{H}_{8}, 2.5 \% \mathrm{H}_{2}$ balanced with $\mathrm{N}_{2}$. The total flow rate of the reactant mixture was $200 \mathrm{~cm}^{3} \mathrm{~min}^{-1}$. After $2 \mathrm{~min}$ on-stream, the catalyst selectivity was compared below $20 \%$ conversion at $550{ }^{\circ} \mathrm{C}$ and turnover rates (TORs, per surface Pt site) were measured under differential condition at conversion below $5 \%$. For iso-butane dehydrogenation, a reaction atmosphere of $2.5 \% \mathrm{C}_{3} \mathrm{H}_{8}, 2.5 \% \mathrm{H}_{2}$ balanced in $\mathrm{N}_{2}$ with a total flow rate of $100 \mathrm{~cm}^{3} \mathrm{~min}^{-1}$ was used. Catalyst performance was measured at $450^{\circ} \mathrm{C}$.

\section{Data availability}

The datasets generated during and/or analyzed during the current study are available from the corresponding authors on reasonable request.

Received: 7 July 2018 Accepted: 2 November 2018

Published online: 10 December 2018

\section{References}

1. Bell, A. T. The impact of nanoscience on heterogeneous catalysis. Science 299, 1688-1691 (2003).

2. Shekhar, M. et al. Size and support effects for the water-gas shift catalysis over gold nanoparticles supported on model $\mathrm{Al}_{2} \mathrm{O}_{3}$ and $\mathrm{TiO}_{2}$. J. Am. Chem. Soc. 134, 4700-4708 (2012).

3. Huang, $\mathrm{X}$. et al. High-performance transition metal-doped $\mathrm{Pt}_{3} \mathrm{Ni}$ octahedra for oxygen reduction reaction. Science 348, 1230-1234 (2015).

4. Matsubu, J. C. et al. Adsorbate-mediated strong metal-support interactions in oxide-supported Rh catalysts. Nat. Chem. 9, 120 (2017).

5. Kattel, S., Liu, P. \& Chen, J. G. Tuning selectivity of $\mathrm{CO}_{2}$ hydrogenation reactions at the metal/oxide interface. J. Am. Chem. Soc. 139, 9739-9754 (2017).

6. Zhao, Z.-J. et al. Importance of metal-oxide interfaces in heterogeneous catalysis: a combined DFT, microkinetic, and experimental study of water-gas shift on $\mathrm{Au} / \mathrm{MgO}$. J. Catal. 345, 157-169 (2017).

7. Mehta, P., Greeley, J., Delgass, W. N. \& Schneider, W. F. Adsorption energy correlations at the metal-support boundary. ACS Catal. 7, 4707-4715 (2017)

8. $\mathrm{Li}, \mathrm{W}$.-Z. et al. Stable platinum nanoparticles on specific $\mathrm{MgAl}_{2} \mathrm{O}_{4}$ spinel facets at high temperatures in oxidizing atmospheres. Nat. Commun. 4, 2481 (2013).

9. Rao, R. G. et al. Interfacial charge distributions in carbon-supported palladium catalysts. Nat. Commun. 8, 340 (2017).

10. Cargnello, M. et al. Control of metal nanocrystal size reveals metal-support interface role for ceria catalysts. Science 341, 771-773 (2013).

11. Willinger, M. G. et al. A case of strong metal-support interactions: combining advanced microscopy and model systems to elucidate the atomic structure of interfaces. Angew. Chem. Int. Ed. 53, 5998-6001 (2014).

12. Tauster, S., Fung, S. \& Garten, R. L. Strong metal-support interactions. Group 8 noble metals supported on titanium dioxide. J. Am. Chem. Soc. 100, 170-175 (1978).

13. Tauster, S., Fung, S., Baker, R. \& Horsley, J. Strong interactions in supportedmetal catalysts. Science 211, 1121-1125 (1981).

14. Naguib, M. et al. Two-dimensional nanocrystals produced by exfoliation of $\mathrm{Ti}_{3} \mathrm{AlC}_{2}$. Adv. Mater. 23, 4248-4253 (2011).

15. Naguib, M., Mochalin, V. N., Barsoum, M. W. \& Gogotsi, Y. 25th anniversary article: MXenes: a new family of two-dimensional materials. Adv. Mater. 26, 992-1005 (2014).

16. $\mathrm{Li}, \mathrm{Z}$. et al. Reactive metal-support interactions at moderate temperature in two-dimensional niobium-carbide-supported platinum catalysts. Nat. Catal. $\mathbf{1}$, 349-355 (2018)

17. Penner, S. \& Armbrüster, M. Formation of intermetallic compounds by reactive metal-support interaction: a frequently encountered phenomenon in catalysis. ChemCatChem 7, 374-392 (2015).

18. Bernal, S. et al. Nanostructural Evolution of a $\mathrm{Pt} / \mathrm{CeO}_{2}$ catalyst reduced at Increasing temperatures (473-1223 K): a HREM study. J. Catal. 169, 510-515 (1997).

19. Beard, B. C. \& Ross, P. N. Platinum-titanium alloy formation from hightemperature reduction of a titania-impregnated platinum catalyst: implications for strong metal-support interaction. J. Phys. Chem. 90, 6811-6817 (1986)

20. Sabnis, K. D. et al. Water-gas shift catalysis over transition metals supported on molybdenum carbide. J. Catal. 331, 162-171 (2015).

21. Sattler, J. J., Ruiz-Martinez, J., Santillan-Jimenez, E. \& Weckhuysen, B. M. Catalytic dehydrogenation of light alkanes on metals and metal oxides. Chem. Rev. 114, 10613-10653 (2014).

22. Cui, Z. et al. Synthesis of structurally ordered $\mathrm{Pt}_{3} \mathrm{Ti}$ and $\mathrm{Pt}_{3} \mathrm{~V}$ nanoparticles as methanol oxidation catalysts. J. Am. Chem. Soc. 136, 10206-10209 (2014).

23. Saravanan, G. et al. $\mathrm{Pt}_{3} \mathrm{Ti}$ nanoparticles: fine dispersion on $\mathrm{SiO}_{2}$ supports, enhanced catalytic $\mathrm{CO}$ oxidation, and chemical stability at elevated temperatures. Langmuir 26, 11446-11451 (2010).

24. Naguib, M. et al. New two-dimensional niobium and vanadium carbides as promising materials for Li-ion batteries. J. Am. Chem. Soc. 135, 15966-15969 (2013).

25. Mashtalir, O. et al. Intercalation and delamination of layered carbides and carbonitrides. Nat. Commun. 4, 1716 (2013).

26. Lin, H., Gao, S., Dai, C., Chen, Y. \& Shi, J. A Two-dimensional biodegradable niobium carbide (MXene) for photothermal tumor eradication in NIR-I and NIR-II biowindows. J. Am. Chem. Soc. 139, 16235-16247 (2017).

27. Nawaz, Z. Light alkane dehydrogenation to light olefin technologies: a comprehensive review. Rev. Chem. Eng. 31, 413-436 (2015).

28. Gallagher, J. R. et al. Structural evolution of an intermetallic Pd-Zn catalyst selective for propane dehydrogenation. Phys. Chem. Chem. Phys. 17, 28144-28153 (2015)

29. Wu, Z. et al. Pd-In intermetallic alloy nanoparticles: highly selective ethane dehydrogenation catalysts. Catal. Sci. Technol. 6, 6965-6976 (2016).

30. Wegener, E. C. et al. Structure and reactivity of Pt-In intermetallic alloy nanoparticles: Highly selective catalysts for ethane dehydrogenation. Catal. Today 299, 146-153 (2017). 
31. Hammer, B., Morikawa, Y. \& Nørskov, J. K. CO chemisorption at metal surfaces and overlayers. Phys. Rev. Lett. 76, 2141 (1996).

32. Cybulskis, V. J. et al. Zinc Promotion of platinum for catalytic light alkane dehydrogenation: insights into geometric and electronic effects. ACS Catal. 7, 4173-4181 (2017).

33. Hammer, B. \& Nørskov, J. K. Theoretical surface science and catalysiscalculations and concepts. Adv. Catal. 45, 71-129 (2000).

34. Yu, W., Porosoff, M. D. \& Chen, J. G. Review of Pt-based bimetallic catalysis: from model surfaces to supported catalysts. Chem. Rev. 112, 5780-5817 (2012).

35. Cortright, R. \& Dumesic, J. Microcalorimetric, spectroscopic, and kinetic studies of silica supported $\mathrm{Pt}$ and $\mathrm{Pt} / \mathrm{Sn}$ catalysts for isobutane dehydrogenation. J. Catal. 148, 771-778 (1994).

36. Yang, C. et al. Promotion of $\mathrm{Pd}$ nanoparticles by $\mathrm{Fe}$ and formation of a $\mathrm{Pd}_{3} \mathrm{Fe}$ intermetallic alloy for propane dehydrogenation. Catal. Today, https://doi.org/ 10.1016/j.cattod.2018.07.043 (2018).

37. Yang, M.-L., Zhu, Y.-A., Zhou, X.-G., Sui, Z.-J. \& Chen, D. First-principles calculations of propane dehydrogenation over PtSn catalysts. Acs Catal. 2, 1247-1258 (2012).

38. Yang, M.-L. et al. DFT study of propane dehydrogenation on Pt catalyst: effects of step sites. Phys. Chem. Chem. Phys. 13, 3257-3267 (2011).

\section{Acknowledgements}

Y. W. appreciates the support from the Herbert L. Stiles Professorship and Iowa State University College of Engineering exploratory research projects. Z.W., and J.T.M., were supported in part by the National Science Foundation under Cooperative Agreement No. EEC-1647722, and W.N.D. by the Davidson School of Chemical Engineering at Purdue. The XPS data was obtained at the Surface Analysis Facility of the Birck Nanotechnology Center of Purdue University. Use of the Advanced Photon Source was supported by the U.S. Department of Energy, Office of Basic Energy Sciences, under contract no. DEAC02-06CH11357. MRCAT operations, beamline 10-BM, are supported by the Department of Energy and the MRCAT member institutions. L.Y. and H.X. acknowledge financial support from the American Chemical Society Petroleum Research Fund (ACS PRF 55581-DNI5) and the NSF CBET Catalysis Program (CBET-1604984). The computational resource used in this work is provided by the advanced research computing at Virginia Polytechnic Institute and State University.

\section{Author contributions}

Z.L. and Z.W. conceived the idea and designed the present work. H.X., L.Y., and J. L. conducted DFT calculations. Z.L., Z.W., Y.C., N.L., and Z.Q. synthesized the catalysts and performed the catalytic evaluation. C.M., Z.W., J.M., carried out the spectroscopic characterizations. L.Z., T.M., Z.L., B.X., and E.Shi performed the detailed microscopic experiments. Y.W., J.M., H.X., and W.N.D. supervised the research.

\section{Additional information}

Supplementary Information accompanies this paper at https://doi.org/10.1038/s41467018-07502-5.

Competing interests: Provisional patent applications have been applied through the Iowa State University (US. Provisional Patent Application 62/572,903.).

Reprints and permission information is available online at http://npg.nature.com/ reprintsandpermissions/

Publisher's note: Springer Nature remains neutral with regard to jurisdictional claims in published maps and institutional affiliations.

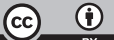

Open Access This article is licensed under a Creative Commons Attribution 4.0 International License, which permits use, sharing, adaptation, distribution and reproduction in any medium or format, as long as you give appropriate credit to the original author(s) and the source, provide a link to the Creative Commons license, and indicate if changes were made. The images or other third party material in this article are included in the article's Creative Commons license, unless indicated otherwise in a credit line to the material. If material is not included in the article's Creative Commons license and your intended use is not permitted by statutory regulation or exceeds the permitted use, you will need to obtain permission directly from the copyright holder. To view a copy of this license, visit http://creativecommons.org/ licenses/by/4.0/.

(C) The Author(s) 2018 